\title{
The effect of consolidated periods in heterogeneous lot- sizing games
}

\author{
Luis A. Guardiola ${ }^{1}$ (D) Ana Meca $^{2}$ (D) $\cdot$ Justo Puerto $^{3}$ (D)
}

Received: 6 September 2020 / Accepted: 8 July 2021 / Published online: 9 September 2021

(C) The Author(s) 2021

\begin{abstract}
We consider a cooperative game defined by an economic lot-sizing problem with heterogeneous costs over a finite time horizon, in which each firm faces demand for a single product in each period and coalitions can pool orders. The model of cooperation works as follows: ordering channels and holding and backlogging technologies are shared among the members of the coalitions. This implies that each firm uses the best ordering channel and holding technology provided by the participants in the consortium. That is, they produce, hold inventory, pay backlogged demand and make orders at the minimum cost of the coalition members. Thus, firms aim at satisfying their demand over the planing horizon with minimal operation cost. Our contribution is to show that there exist fair allocations of the overall operation cost among the firms so that no group of agents profit from leaving the consortium. Then we propose a parametric family of cost allocations and provide sufficient conditions for this to be a stable family against coalitional defections of firms. Finally, we focus on those periods of the time horizon that are consolidated and we analyze their effect on the stability of cost allocations.
\end{abstract}

Keywords Cost-sharing · Lot-sizing $\cdot$ Heterogeneous costs $\cdot$ Consolidated period - Cooperative game

Luis A. Guardiola

luis.guardiola@ua.es

Ana Meca

ana.meca@umh.es

Justo Puerto

puerto@us.es

1 Departamento de Fundamentos del Análisis Económico, Universidad de Alicante, 03071 Alicante, Spain

2 I.U. Centro de Investigación Operativa, Universidad Miguel Hernández, Edificio Torretamarit, Avda. de la Universidad s.n., 03202 Elche, Alicante, Spain

3 Mathematical Research Institute of the University of Seville (IMUS), 41012 Sevilla, Spain 
Mathematics Subject Classification 91A12 $90 \mathrm{~B} 05$

\section{Introduction}

Lot-sizing is one of the most important and also one of the most difficult problems in production planning. Although lot-sizing and scheduling problems, and their variants, have been studied by many authors, providing different solution approaches, looking for more efficient solution approaches is still a challenging subject. Variants of the lot-sizing problem (henceforth, LSP) with complex setup and other variants which are more realistic and practical have received less attention in the literature. There has been little literature regarding problems such as LSP with backlogging or with setup times and setup carry-over. Since these problems are NPhard, fast and efficient heuristics are required. A good survey on the subject is Karimi et al. (2003).

Also there is little literature for cost-sharing in LSP. Among the pioneers we mention Van den Heuvel et al. (2007) which focuses on the cooperation in economic lot-sizing situations (henceforth ELS-situations) with homogeneous costs. They consider a homogeneous finite horizon model where cost are all equal for all the players in each period. Each player must satisfy its demand in each period by producing or carrying inventory from previous stages but backlogging is not permitted. The main result in that paper is that the cooperative games induced by ELS-situations enjoy a nonempty core. Subsequently, (Guardiola et al. 2008, 2009, 2021b) present a new class of totally balanced combinatorial optimization games: production-inventory games (henceforth, PI-games). PI-games bring the essentials of inventory and combinatorial optimization games. They provide a cooperative approach to analyze the production and storage of indivisible items being their characteristic function given as the optimal objective function of a combinatorial optimization problem. PI-games can be seen as ELS games without setup costs but with backlogging. (Guardiola et al. 2008, 2009) prove that the Owen set, the set of allocations which are achievable through dual solutions [see Owen (1975) and Gellekom et al. (2000)] reduces to a singleton. In addition, that allocation is always in the core and it defines a population monotonic allocation scheme. This fact motivates the name of Owen point for this core-allocation on PIgames. Recently, Guardiola et al. (2021a) introduce new core allocations for PIgames improving the weaknesses of the Owen point (it may be seen as an altruistic allocation for any players) and study the structure and complexity of the core of PIgames. The main difference between the ELS-games by Van den Heuvel et al. (2007) and PI-games by Guardiola et al. (2009) is that the former considers set up costs but assume that costs are homogeneous for all players in each period. Hence, both situations (ELS and PI) are, in general, different.

On the other hand, $\mathrm{Xu}$ and Yang (2009) present a cost-sharing method that is competitive, cross-monotonic and approximate cost recovering for an ELS-game under a weak triangle inequality assumption, along with numerical results showing the effectiveness of the proposed method. Li et al. (2014) present a cost-sharing 
method that is cross-monotonic, competitive, and approximate cost recovery, for the multi-level ELS-game, under a mild condition. This result extends that of ELSgame of $\mathrm{Xu}$ and Yang (2009).

Dreschel (2010) focusses on cooperative lot-sizing games in supply chains. Several models of cooperation in lot-sizing problems of different complexity that are analyzed regarding theoretical properties like monotonicity or concavity and solved with the proposed row generation algorithm to compute core elements; i.e., determining stable and fair cost allocations.

In another paper, Gopaladesikan and Uhan (2011) consider a new class of cooperative cost-sharing games, associated with the ELS-problem with remanufacturing options (henceforth, ELSR). They investigate the relative strength and integrality gaps of various mathematical optimization formulations of ELSR. Using insights from these results, they study the core of the associated cost-sharing game and show it is empty, in general. However, for two special cases-zero setup costs, and large initial quantity of low cost returns - they find that the cost sharing game has a non-empty core, and that a cost allocation in the core can be computed in polynomial time.

Zeng et al. (2011) consider the ELS-game with perishable inventory. In this cooperative game, a number of retailers that have a known demand through a fixed number of periods for a same kind of perishable goods collaborate to place joint orders to a single supplier. They first show that an ELS game with perishable inventory is subadditive, totally balanced and its core is non-empty. Then, they propose a core-allocation which allocates the unit cost to each period as equally as possible. Finally, a numerical example is given to illustrate the above results.

Tsao et al. (2013) use the Nash game and the cooperation game in an imperfect production system to investigate the combined effects of lot-sizing integration, learning effect, and an imperfect production process on a manufacturer-retailer channel. They also developed a search procedure to solve the problem described, the optimal properties and a numerical study are conducted to seek for structural and quantitative insights into the relationship between the upstream and downstream entities of the supply chain. Numerical results indicated that the cooperation game policy created a higher cost reduction under a wide range of parameter settings.

Chen and Zhang (2016) consider the ELS-game with general concave ordering cost. In that paper, the dual variables are understood as the price of the demand per unit. They show that a core allocation can be computed in polynomial time under the assumption that all retailers have the same cost parameters (again homogeneous costs). Their approach is based on linear programming (LP) duality. Specifically, they prove that there exists an optimal dual solution that defines an allocation in the core and point out that it is not necessarily true that every optimal dual solution defines a core allocation. Toriello and Uhan (2014) also study ELS-games with general concave ordering costs and show how to compute a dynamic cost allocation in the strong sequential core of these games, i.e., an allocation over time that exactly distributes costs and is stable against coalitional defections at every period of the time horizon.

More recent papers that are related to ELS-games are Masheli and Mohammaditabar (2017), Margaretha and Hartl (2020), and Feng et al. (2021). In Masheli and 
Mohammaditabar (2017) studies a supplier selection model. The joint decision making of procurement lot-size, supplier selection, production decisions and shipment policy selection has potential to reduce total supply chain costs. The authors present a single-buyer multi-suppliers model in a two level supply chain and a cooperative game theory model is proposed to analyse the decisions. The selected suppliers and total supply chain costs are found. The authors show that the cooperative model could result in a stable solution with same total supply chain cost as the centralized model and also, when suppliers have equal opportunity costs for each single production capacity, selected suppliers are determined independent from the opportunity cost but when the suppliers have different opportunity costs, the selected suppliers are influenced by the opportunity cost that they have. A numerical example describes the findings. Margaretha and Hartl (2020) introduce the collaborative multi-level lot-sizing problem with cost synergies. This arises if producers can realise reductions of their costs by providing more than one product in a specific time horizon. The authors propose a decentralised mechanism, where producers do not have to reveal their individual items costs. Additionally, a Genetic Algorithms-based centralised approach is developed, which we use for benchmarking. Their study shows that this approach comes very close to the a central plan, while in the decentralised one no critical information has to be shared. Finally, Feng et al. (2021) profit allocation rules for joint replenishment among retailers under a carbon cap-and-trade policy. They show that joint replenishment can increase participants' total profit and reduce their total amount of carbon emissions. To allocate the total profit from joint replenishment to the retailers, a joint replenishment game with the carbon cap-and-trade policy is introduced. It turns out this game is convex. Based on this, they design an altruistic profit core allocation rule by categorizing retailers into efficient ones and non-efficient ones, with the efficient retailers being altruistic who will transfer their surplus carbon allowance to those non-efficient ones. Moreover, they get results concerning how much carbon allowance, to whom and at what price retailers are will- ing to transfer in the grand coalition, by considering their altruistic parameters. Their results show that the retailer with the highest altruistic parameter value obtains all the surplus carbon allowance from other retailers, and this particular retailer transfers his surplus carbon allowance to the retailer with the second-highest altruistic parameter value. These results indicate that people do not help other people uniformly; rather, they do so according to the generosity of other people.

In this paper, we study another class of totally balanced combinatorial optimization games called setup-inventory games (henceforth, SI-games) that arises from cooperation in lot-sizing problems with heterogeneous costs. Each firm faces demand for a single product in each period and coalitions can pool orders. Firms cooperate which implies that each firm uses the best ordering channel and holding technology provided by the participants in the consortium. That is, they produce, hold inventory, pay backlogged demand and make orders at the minimum cost among the members of the coalition. Thus, firms aim at satisfying their demand over the planing horizon with minimal operation cost. In principle sharing private information can be seem as a limitation of this model. However, the reader may notice that this can be easily overcome. To prevent disclosing private information 
one can assume that firms communicate via a mediator who attempts to streamline their decisions without restricting their freedom. The mediator implements the cooperation mechanism without disclosing information, reaching a win-win situation for all involved entities and giving rise to acceptable costs allocations.

Therefore, each firm must solve the Wagner and Whitin extended model with backlogging costs, solved by Zangwill (1969) using dynamic programming techniques. Modeling cooperation in production and holding costs has already appeared in literature. Additionally, our cooperation in backlogging is also natural, but new: all the members of a coalition pay backlogging cost (compensation for delayed demand) by the cheapest cost among those in the coalition. The strength of the coalitions increase with their size so that larger coalitions can get better opportunities in the market. To illustrate this form of cooperation today, let us consider four automotive companies, Peugeot (P), Citroen (C), Fiat (F) and Iveco (I). They all use the same chassis for their cars and buy it from a Chinese supplier twice a year ( 2 periods). Peugeot is interested in buying a larger quantity of chassis to avoid supply problems from China and a possible increase in transportation costs. In addition, $\mathrm{P}$ is able to negotiate with the Chinese supplier and get very competitive unit and period production costs for large order sizes. $\mathrm{P}$ then proposes to the other companies to place a joint order for chassis. Citroen thinks this is a good idea because it has a large warehouse in Vigo where the entire order can be always stored. Iveco proposes to take advantage of its good contacts with transport companies and to hire a ship to transport the joint orders from China to Vigo. The fixed order and transport costs are included in the set up costs. Fiat analyzes the proposal and, although it does not have a long delay per period in the delivery of its cars, and its penalty costs are the lowest, it concludes that it turns out profitable. The four companies then agree and taking the unit production costs of $\mathrm{P}$, the holding costs of $\mathrm{C}$, the setup costs of $\mathrm{I}$ and the backlogging costs of $\mathrm{F}$, they place a joint order with the lowest total cost $\mathrm{c}(\mathrm{N})$. We should note that the cooperation of the four companies generates a reduction in the backlogging costs of $\mathrm{P}, \mathrm{C}$ and I (to those of F) and thus it is profitable for the consortium.

SI-games are an extension of PI-games (Guardiola et al. 2009) since the latter do not include setup costs. The reader may note that whenever set up costs are zero in all periods SI-games become PI-games. Moreover, SI-games also generalize ELSgames in that all considered costs can be different for the different players in each period and, in addition, backorders are permitted.

The contribution of this paper is to prove that the above mentioned mode of cooperation is always stable in that there exist fair allocations of the overall cost of the system among the members of a coalition so that no subgroup of agents is better off by leaving the consortium (every SI-game has a nonempty core). Then we propose a parametric family of cost allocations for SI-games: the extended Owen points. We provide sufficient conditions for this to be a stable family against coalitional defections of firms, that is, every extended Owen point is a coreallocation. Finally, we focus on those periods of the time horizon that are consolidated and analyze their effect on the stability of cost allocations. Specifically we prove that for consolidated SI-situations, the single extended Owen point belongs to the core of the game. Our paper contributes as well to the literature on the 
analysis of problems in Operations Research by means of cooperative games. The interested reader is referred to Borm et al. (2001) for further details on operations research games (including inventory games).

\section{Model}

We start by describing the basic form of lot-sizing problems (see Johnson and Montgomery (1974) for further details). We focus here on periodic review inventory problems where a setup cost is incurred when placing an order, which in turns makes the cost structure non-linear.

A setup-inventory problem (hereafter SI-problem) can be described as follows. We consider $T$ periods, numbered from 1 to $T$, where the demand for a single product occurs in each of them. This demand is satisfied by own production, and can be done in three different periods: (i) the same period, (ii) an earlier period (as inventory), (iii) a later period (as backlogging). In each production period, a fixed cost must be paid. Therefore, the model includes production, inventory holding, backlogging and setup costs. We assume, without loss of generality, the initial and terminal inventories are set to zero. The objective is to find an optimal ordering plan, that is a feasible ordering plan that minimizes the sum of setup, production, inventory holding and backlogging cost.

For each period $t=1, \ldots, T$ we let:

$-\quad d_{t}:=$ demand in period $t$ and $d=\left(d_{1}, \ldots, d_{T}\right)$.

$-\quad k_{t}:=$ setup cost in period $t$ and $k=\left(k_{1}, \ldots, k_{T}\right)$.

- $h_{t}:=$ unit inventory carrying costs in period $t$ and $h=\left(h_{1}, \ldots, h_{T}\right)$.

$-b_{t}:=$ unit backlogging carrying costs in period $t$ and $b=\left(b_{1}, \ldots, b_{T}\right)$.

$-p_{t}:=$ unit production costs in period $t$ and $p=\left(p_{1}, \ldots, p_{T}\right)$.

$-q_{t}:=$ order size in period $t$.

- $I_{t}:=$ ending inventory in period $t$.

$-E_{t}:=$ ending backlogged demand in period $t$.

We consider that costs and demand can never be negative. Furthermore, the decision variables $q_{t}, I_{t}$ and $E_{t}$ take integer values. The single-item formulation of the problem $(T, d, k, h, b, p)$ is as follows:

$$
\begin{aligned}
(S I) \quad & \min \sum_{t=1}^{T}\left[p_{t} q_{t}+h_{t} I_{t}+b_{t} E_{t}+k_{t} \gamma\left(q_{t}\right)\right] \\
& \text { s.t. } I_{0}=I_{T}=E_{0}=E_{T}=0, \\
& I_{t}-E_{t}=I_{t-1}-E_{t-1}+q_{t}-d_{t}, \quad t=1, \ldots, T, \\
& q_{t}, I_{t}, E_{t}, \text { non-negative, integer, } t=1 \ldots, T,
\end{aligned}
$$

where, 


$$
\gamma(q):= \begin{cases}1 & \text { if } q>0 \\ 0 & \text { if } q=0 .\end{cases}
$$

Zangwill (1969) proved that there exists an optimal solution to $(S I)$ such that, for each $t=1, \ldots, T, E_{t}>0$ and $I_{t}>0$ simultaneously; i.e., it is not optimal to divide the production to satisfy demand over multiple periods. We define a feasible ordering plan for a SI-problem as $\sigma \in \mathbb{R}^{T}$ where $\sigma_{t} \in T \cup\{0\}$ denotes the period where demand of period $t$ is ordered. We assume the convention that $\sigma_{t}=0$ if and only if $d_{t}=0$. It means that there is no order placed to satisfy a null demand at period t. Any ordering plan $\sigma$ with $\sigma \in \mathbb{R} \%^{T}$ and $\sigma_{t} \in T \cup\{0\}\left(\sigma_{t}=\right.$ 0 if and only if $\left.d_{t}=0\right)$ is implementable since it would be a production plan that firms could carry it out. A feasible ordering plan must be a production plan that meets all the requirements on satisfying production and demand. For instance, if $\sigma_{t}=0$ for all periods $t$ and there is some positive demand, then this plan would not be feasible since it would violate the condition of the optimization problem (SI) that requires satisfying the demand of all the periods. Moreover, we define $P(\sigma) \in \mathbb{R}^{T}$ as the cost vector associated to ordering plan $\sigma$ (henceforth: cost-plan vector), where

$$
P_{t}(\sigma):=\left\{\begin{array}{ccc}
0 & \text { if } & \sigma_{t}=0, \\
p_{t}, & \text { if } & \sigma_{t}=t, \\
p_{\sigma_{t}}+\sum_{r=\sigma_{t}}^{t-1} h_{r}, & \text { if } & 1 \leq \sigma_{t}<t \\
p_{\sigma_{t}}+\sum_{r=t+1}^{\sigma_{t}} b_{r}, & \text { if } & t<\sigma_{t} \leq T .
\end{array}\right.
$$

If $\sigma^{*}$ is an optimal ordering plan for a SI-problem, then the optimal cost is given by

$$
v(S I)=P\left(\sigma^{*}\right)^{\prime} d+\delta\left(\sigma^{*}\right)^{\prime} k=\sum_{t=1}^{T}\left(P_{t}\left(\sigma^{*}\right) d_{t}+\delta_{t}\left(\sigma^{*}\right) k_{t}\right),
$$

where, $\delta\left(\sigma^{*}\right)=\left(\delta_{t}\left(\sigma^{*}\right)\right)_{t \in T}$ and

$$
\delta_{t}\left(\sigma^{*}\right):=\left\{\begin{array}{cc}
1 & \text { if } \exists r \in T: \sigma_{r}^{*}=t \\
0 & \text { otherwise. }
\end{array}\right.
$$

Notice that if all setup costs are zero, the problem we are dealing with is a PIproblem (see Guardiola et al. 2009, 2021b). Moreover, although the model assumes that companies produce their demand, we can interchangeably consider the case where demand is satisfied either by producing or purchasing. One has simply to interpret that the purchasing costs can be ordering costs (set up costs) and unit purchasing costs (variable costs).

\section{Cooperation in lot-sizing with heterogeneous costs}

Next we address a variant of this model where several firms, facing each one a SIproblem, coordinate their actions to reduce costs. This coordination is driven by sharing ordering channels, backlogged and inventory carrying technologies. This means that cooperating firms make a joint order and pay backlogged and inventory 
carrying demand at the cheapest costs among the members of the coalition at each period. Formally, a setup-inventory situation (henceforth, SI-situation) is a tuple $(N, D, K, H, B, P)$ where:

- $N=\{1, \ldots, n\}$ is the set of players.

- $D=\left[d^{1}, \ldots, d^{n}\right]^{\prime}$ is an integer demand matrix, where each row corresponds to the demand of a player, that is, $d^{i}=\left[d_{1}^{i}, \ldots, d_{T}^{i}\right]^{\prime}$.

- $K=\left[k^{1}, \ldots, k^{n}\right]^{\prime}$ is a setup cost matrix, where $k^{i}=\left[k_{1}^{i}, \ldots, k_{T}^{i}\right]^{\prime}$.

- $H=\left[h^{1}, \ldots, h^{n}\right]^{\prime}$ is an inventory carrying costs matrix, where $h^{i}=\left[h_{1}^{i}, \ldots, h_{T}^{i}\right]^{\prime}$.

- $B=\left[b^{1}, \ldots, b^{n}\right]^{\prime}$ is a backlogging carrying costs matrix, where $b^{i}=\left[b_{1}^{i}, \ldots, b_{T}^{i}\right]^{\prime}$.

- $P=\left[p^{1}, \ldots, p^{n}\right]^{\prime}$ is a production costs matrix, where $p^{i}=\left[p_{1}^{i}, \ldots, p_{T}^{i}\right]^{\prime}$.

To simplify the notation, we define $Z$ as a matrix in which all costs are included, that is, $Z:=(K, H, B, P)$. A cost TU-game is a pair $(N, c)$, where $N$ is the finite player set, $\mathcal{P}(N)$ is the power set of $\mathrm{N}$ (this is to say the set of all coalitions of $\mathrm{N}$ ) and $c: \mathcal{P}(N) \rightarrow \mathbb{R}$ the characteristic function satisfying $c(\varnothing)=0$. The subgame related to coalition $S, c_{S}$, is the restriction of the mapping $c$ to the subcoalitions of $S$. A cost allocation will be $x \in \mathbb{R}^{n}$ and, for every coalition $S \subseteq N$ we shall write $x(S):=$ $\sum_{i \in S} x_{i}$ the cost-sharing to coalition $S$ (where $x(\varnothing)=0$ ).

For each SI-situation $(N, D, Z)$, we associate a cost TU-game $(N, c)$ where, for any nonempty coalition $S \subseteq N, c(S)$ is the optimal value of the optimization problem $S I(S)$, defined as:

$$
\begin{aligned}
(S I(S)) \quad & \min \sum_{t=1}^{T}\left(p_{t}^{S} q_{t}+h_{t}^{S} I_{t}+b_{t}^{S} E_{t}+k_{t}^{S} \gamma\left(q_{t}\right)\right) \\
& \text { s.t. } I_{0}=I_{T}=E_{0}=E_{T}=0 \\
& I_{t}-E_{t}=I_{t-1}-E_{t-1}+q_{t}-d_{t}^{S}, \quad t=1, \ldots, T, \\
& q_{t} \geq 0, I_{t} \geq 0, E_{t} \geq 0, \text { and integers, } t=1, \ldots, T ;
\end{aligned}
$$

with

$$
p_{t}^{S}:=\min _{i \in S}\left\{p_{t}^{i}\right\}, h_{t}^{S}:=\min _{i \in S}\left\{h_{t}^{i}\right\}, b_{t}^{S}:=\min _{i \in S}\left\{b_{t}^{i}\right\}, k_{t}^{S}:=\min _{i \in S}\left\{k_{t}^{i}\right\}, d_{t}^{S}:=\sum_{i \in S} d_{t}^{i} .
$$

Notice that for all nonempty $S \subseteq N$ the characteristic function $c$ can be rewritten as follows:

$$
c(S)=P^{S}\left(\sigma^{S}\right)^{\prime} d^{S}+\delta\left(\sigma^{S}\right)^{\prime} k^{S}=\sum_{t=1}^{T}\left(P_{t}^{S}\left(\sigma^{S}\right) d_{t}^{S}+\delta_{t}\left(\sigma^{S}\right) k_{t}^{S}\right),
$$

where $\sigma^{S}, P_{t}^{S}\left(\sigma^{S}\right) \in \mathbb{R}^{T}$ are the optimal ordering plan and the cost-plan vector associated to $S I(S)$, respectively. Every cost TU-game defined in this way is what we call a setup-inventory game (SI-game).

The reader may notice that every PI-game (as introduced by Guardiola et al. 2009 ) is a SI-game with $K=0$. Hence the class of PI-games is a particular subclass of the SI-games. 
First, we wonder whether the above model of cooperation is stable, i.e., whether there is a fair division of the total cost among the players such that no group of them has incentives to leave. As we had already announced the concept of core provides a direct answer to that question. The core of the game $(N, c)$ consists of those cost allocations which divide the cost of the grand coalition in such a way that any other coalition pays at most its cost by the characteristic function. Formally,

$\operatorname{Core}(N, c):=\left\{x \in \mathbb{R}^{n}: x(N)=c(N)\right.$ and $x(S) \leq c(S)$ for all $\left.S \subset N\right\}$.

In the following, fair allocations of the total cost will be called core-allocations.

Bondareva (1963) and Shapley (1967) independently provide a general characterization of games with a non-empty core by means of balanceness. A collection of coalitions in $N, \mathcal{B} \subseteq \mathcal{P}(N)$ is a balanced collection if there exist nonnegative weights $\left\{\lambda_{S}\right\}_{S \in \mathcal{B}}$ such that $\sum_{S \in \mathcal{B}: i \in S} \lambda_{S}=1$ for all $i \in N$. Those weights $\left\{\lambda_{S}\right\}_{S \in \mathcal{B}}$ are called balancing coefficients. A cost game $(N, c)$ is balanced if for every balanced collection $\mathcal{B}$ with balancing coefficients $\left\{\lambda_{S}\right\}_{S \in \mathcal{B}}$ it holds that

$$
\sum_{S \in \mathcal{B}} \lambda_{S} c(S) \geq c(N)
$$

Then, Bondareva and Shapley prove that $(N, c)$ has a nonempty core if and only if it is balanced. In addition, it is totally balanced game if the core of every subgame is nonempty. Totally balanced games were introduced by Shapley and Shubik in the study of market games (see Shapley and Shubik 1969).

It is important to remark that even though the problems that define SI-games have totally unimodular constraint matrices, dual solutions do not induce core solution throughout the Owen construction. The reason is because the mixed integer linear programming reformulation of $S I(S)$ does not have a strong dual (Recall that a pair of dual problems without duality gap are said to be strong dual (Tamir 1992)). Therefore, the question whether the core of the corresponding game is empty or not is a challenging query and its study makes sense.

The main result of this section states that the cooperation in lot-sizing problems with heterogeneous costs is always stable. In other words, SI-games are balanced. In what follows we include a technical lemma that helps in proving the following theorem. Actually, it provides a procedure for constructing new ordering plans out of existing ones. The rationale behind this construction is similar to the one used in Van den Heuvel et al. (2007).

Lemma 1 Let $\mathcal{B}$ be a balanced collection of coalitions with balancing coefficients $\left\{\lambda_{S}\right\}_{S \in \mathcal{B}}$. Assume that for each coalition $S \in \mathcal{B}, \pi^{S}$ is the optimal order plan for the problem $\left(T, d^{S}, k^{N}, h^{N}, b^{N}, p^{N}\right)$. Let $r$ be the smallest positive integer such that $l_{S}$ : $=r \lambda_{S}^{*} \in \mathbb{Z}^{+}$for all $S \in \mathcal{B}$ where $\left\{\lambda_{S}^{*}\right\}_{S \in \mathcal{B}}$ are irreducible rational numbers bounded above by 1 and greater than or equal to $\left\{\lambda_{S}\right\}_{S \in \mathcal{B}}$ (Recall that irreducible refers to numeric fractions that cannot be simplified, that is, fractions such that the numerator and the denominator are coprime). Then, there exist $\left\{\psi^{j}\right\}_{j=1 \ldots, r}$ feasible order plans for the problem $\left(T, d^{N}, k^{N}, h^{N}, b^{N}, p^{N}\right)$ satisfying (i), (ii) and (iii):

(i) For each $t$, there exists $S(j) \in \mathcal{B}$ such that $\psi_{t}^{j}=\left\{\begin{array}{cl}\pi_{t}^{S(j)} & \text { if } d_{t}^{N}>0, \\ 0 & \text { otherwise. }\end{array}\right.$ 
(ii) $S(j)$ is used at most $r \lambda_{S(j)}^{*}$ times $\forall j=1, \ldots, r$.

(iii) For any $j=1, \ldots, r, P_{t}^{N}\left(\pi^{S(j)}\right) \leq P_{t}^{N}\left(\pi^{S}\right)$ for all $S \neq S(i), \forall i=1, \ldots, r$.

Proof The proof of this lemma is constructive so that at the end we will have a procedure to actually construct the corresponding order policies $\left\{\psi^{j}\right\}_{j=1 \ldots, r}$.

Let $\mathcal{B}$ be a balanced collection and $\left\{\lambda_{S}\right\}_{S \in \mathcal{B}}$ their corresponding balancing coefficients. Take $\lambda_{S}^{*}$ as a rational number greater than or equal to $\lambda_{S}$ for all $S \in \mathcal{B}$. There exists $r \in \mathbb{N}$ being the smallest positive integer such that $l_{S}=r \lambda_{S}^{*} \in \mathbb{Z}^{+}$for all $S \in \mathcal{B}$. Notice that this number $r$ satisfies

$$
m:=\sum_{S \in \mathcal{B}} r \lambda_{S}^{*} \geq r
$$

since $\sum_{S \in \mathcal{B}} \lambda_{S}^{*} \geq \sum_{S \in \mathcal{B}} \lambda_{S} \geq 1$.

Consider the following artificial set of coalitions, namely $\mathcal{B A}$. For each coalition $S \in \mathcal{B}$, consider $l_{S}=r \lambda_{S}^{*}$ replicas of $S$ in the set $\mathcal{B A}$. Therefore, we have in total $m$ coalitions in the new collection (see (1)). We assume an arbitrary ordering of these coalitions so that we can refer, without confusion, to any coalition, say $S_{j}$, by its index in such a sequence. Moreover, let $\pi^{S_{j}}$ be the optimal order plan corresponding to the problem $\left(T, d^{S_{j}}, k^{N}, h^{N}, b^{N}, p^{N}\right)$, for each $j=1, \ldots, m$.

Then we proceed to construct the feasible order plans $\left\{\psi^{j}\right\}$. For each period $t$, $1 \leq t \leq T$ set:

i) $\psi_{t}^{j}=0$ for all $j=1, \ldots, r$ if $d_{t}^{N}=0$.

ii) If $d_{t}^{N}>0$ do the following. Define

$$
\begin{aligned}
N_{t}^{*} & =\left\{i \in N: d_{t}^{i}>0\right\}, \\
\mathcal{C}_{t} & =\left\{S_{j}: N_{t}^{*} \cap S_{j} \neq \emptyset, j=1, \ldots, m\right\} .
\end{aligned}
$$

Notice that $N_{t}^{*} \neq \emptyset$ and moreover $\{i\} \subset N_{t}^{*}$ since there exists always at least one agent $i \in N$ such that $d_{t}^{i}>0$, otherwise $d_{t}^{N}=0$. Therefore, $\sum_{S \in \mathcal{B}, i \in S} r \lambda_{S}^{*} \geq r$; and thus $\left|\mathcal{C}_{t}\right| \geq r$. Arrange the coalitions in $\mathcal{C}_{t}$ in nondecreasing sequence, $S_{(1)}, S_{(2)}, \cdots, S_{\left(\left|\mathcal{C}_{t}\right|\right)}$, with respect to the values of $\left\{P_{t}^{N}\left(\pi^{S_{j}}\right)\right\}_{S^{k} \in \mathcal{C}_{t}}$. It is then clear that:

$$
P_{t}^{N}\left(\pi^{S_{(1)}}\right) \leq P_{t}^{N}\left(\pi^{S_{(2)}}\right) \leq \ldots \leq P_{t}^{N}\left(\pi^{S_{\left.\left(\mathcal{C}_{t}\right)\right)}}\right)
$$

Finally, we set $\psi_{t}^{j}=\pi_{t}^{S_{(j)}}$ for $j=1, \ldots, r$. Notice that since $\left|C_{t}\right| \geq r$ this definition is well-stated.

The above construction satisfies the thesis of the lemma.

The following example illustrates how to implement the aforementioned procedure.

Example 1 Consider the following SI-situation with three players and four periods. Notice that all players have the same costs but different demands. 


\begin{tabular}{llllllllllllllllllllll}
\hline & \multicolumn{1}{c}{ Demand } & \multicolumn{4}{c}{ Production } & \multicolumn{4}{c}{ Inventory } & \multicolumn{3}{c}{ Backlogging } & \multicolumn{5}{c}{ Setup } \\
\hline$P 1$ & 1 & 1 & 3 & 2 & 1 & 1 & 2 & 2 & 1 & 2 & 2 & 1 & 1 & 1 & 1 & 1 & 3 & 1 & 1 & 9 \\
$P 2$ & 2 & 1 & 8 & 2 & 1 & 1 & 2 & 2 & 1 & 2 & 2 & 1 & 1 & 1 & 1 & 1 & 3 & 1 & 1 & 9 \\
$P 3$ & 2 & 1 & 9 & 2 & 1 & 1 & 2 & 2 & 1 & 2 & 2 & 1 & 1 & 1 & 1 & 1 & 3 & 1 & 1 & 9 \\
\hline
\end{tabular}

Let $\mathcal{B}:=\{\{1,2\},\{1,3\},\{2,3\},\{1,2,3\}\}$ be a balanced collection of coalitions with balancing coefficients $\left\{\lambda_{S}\right\}_{S \in \mathcal{B}}=\left\{\frac{1}{3}, \frac{1}{3}, \frac{1}{3}, \frac{1}{3}\right\}$. In this case $\left\{\lambda_{S}^{*}\right\}_{S \in \mathcal{B}}=\left\{\lambda_{S}\right\}_{S \in \mathcal{B}}$ and $r=3$.

Next table shows the optimal ordering plans and the corresponding cost plan vectors for the problem $\left(T, d^{S}, k^{N}, h^{N}, b^{N}, p^{N}\right)$ for each coalition $S \in \mathcal{B}$.

\begin{tabular}{lllllllll}
\hline$S$ & $\pi_{1}^{S}$ & $\pi_{2}^{S}$ & $\pi_{3}^{S}$ & $\pi_{4}^{S}$ & $P_{1}^{N}\left(\pi^{S}\right)$ & $P_{2}^{N}\left(\pi^{S}\right)$ & $P_{3}^{N}\left(\pi^{S}\right)$ & $P_{4}^{N}\left(\pi^{S}\right)$ \\
\hline$\{1,2\}$ & 1 & 2 & 3 & 3 & 1 & 1 & 2 & 4 \\
$\{1,3\}$ & 2 & 2 & 3 & 3 & 2 & 1 & 2 & 4 \\
$\{2,3\}$ & 1 & 2 & 3 & 3 & 1 & 1 & 2 & 4 \\
$\{1,2,3\}$ & 1 & 2 & 3 & 4 & 1 & 1 & 2 & 2 \\
\hline
\end{tabular}

The reader may notice that there always exist three feasible order plans for the problem $\left(T, d^{N}, k^{N}, h^{N}, b^{N}, p^{N}\right)$ given by

\begin{tabular}{lllllllll}
\hline$j$ & $\psi_{1}^{j}$ & $\psi_{2}^{j}$ & $\psi_{3}^{j}$ & $\psi_{4}^{j}$ & $P_{1}^{N}\left(\psi^{j}\right)$ & $P_{2}^{N}\left(\psi^{j}\right)$ & $P_{3}^{N}\left(\psi^{j}\right)$ & $P_{4}^{N}\left(\psi^{j}\right)$ \\
\hline 1 & 1 & 2 & 3 & 4 & 1 & 1 & 2 & 2 \\
2 & 1 & 2 & 3 & 3 & 1 & 1 & 2 & 4 \\
3 & 2 & 2 & 3 & 3 & 2 & 1 & 2 & 4 \\
\hline
\end{tabular}

These plans can be built by means of the following recursive procedure: for all $t=1,2,3,4$

- $\psi_{t}^{1}=\pi_{t}^{S(1)}$ such that $S(1)=\arg \min \left\{P_{t}^{N}\left(\pi^{S}\right)\right\}$,

- $\psi_{t}^{2}=\pi_{t}^{S(2)}$ such that $S(2)=\arg \min \left\{P_{t}^{N}\left(\pi^{S}\right): S \neq S(1)\right\}$,

$-\psi_{t}^{3}=\pi_{t}^{S(3)}$ such that $S(3)=\arg \min \left\{P_{t}^{N}\left(\pi^{S}\right): S \neq S(1), S(2)\right\}$,

In addition, for all $S \neq S(1), S(2), S(3), P_{t}^{N}\left(\pi^{S}\right) \geq P_{t}^{N}\left(\pi^{S(j)}\right)=P_{t}^{N}\left(\psi^{j}\right)$, for all $t=$ $1,2,3,4$ and all $j=1,2,3$.

Theorem 1 Every SI-game $(N, c)$ associated to a SI-situation $(N, D, Z)$ is balanced.

Proof Let $\mathcal{B} \subset 2^{N}$ be a balanced collection and $\left\{\lambda_{S}\right\}_{S \in \mathcal{B}}$ their corresponding balancing coefficients. Then, 


$$
\begin{aligned}
\sum_{S \in \mathcal{B}} \lambda_{S} c(S) & =\sum_{S \in \mathcal{B}} \lambda_{S}\left(P^{S}\left(\sigma^{S}\right)^{\prime} d^{S}+\delta\left(\sigma^{S}\right)^{\prime} k^{S}\right) \\
& \geq \sum_{S \in \mathcal{B}} \lambda_{S}\left(P^{N}\left(\sigma^{S}\right)^{\prime} d^{S}+\delta\left(\sigma^{S}\right)^{\prime} k^{N}\right) \\
& \geq \sum_{S \in \mathcal{B}} \lambda_{S}\left(P^{N}\left(\pi^{S}\right)^{\prime} d^{S}+\delta\left(\pi^{S}\right)^{\prime} k^{N}\right),
\end{aligned}
$$

where $\pi^{S}$ for each $S \in \mathcal{B}$ is an optimal order plan for the SI-problem $\left(T, d^{S}, k^{N}, h^{N}, b^{N}, p^{N}\right)$.

Our goal is to prove that

$$
\sum_{S \in \mathcal{B}} \lambda_{S}\left(P^{N}\left(\pi^{S}\right)^{\prime} d^{S}+\delta\left(\pi^{S}\right)^{\prime} k^{N}\right) \geq c(N)
$$

According to the construction in Lemma 1 , take $\lambda_{S}^{\varepsilon_{S}^{S}}$ as a rational number greater than or equal to $\lambda_{S}$ such that

$$
\left\{\begin{array}{lc}
\lambda_{S}^{\varepsilon^{S}}-\lambda_{S}=\varepsilon^{S}=0 & \text { if } \lambda_{S} \text { is rational } \\
\lambda_{S}^{\varepsilon^{S}}-\lambda_{S}=\varepsilon^{S}>0 & \text { otherwise. }
\end{array}\right.
$$

There exists $r \in \mathbb{N}$ being the smallest positive integer for which $r \lambda_{S}^{\varepsilon_{S}^{S}}$ is integer for all $S \in \mathcal{B}$. Then (4) can be rewritten as

$$
\sum_{S \in \mathcal{B}} r \lambda_{S}^{\varepsilon^{S}}\left(P^{N}\left(\pi^{S}\right)^{\prime} d^{S}+\delta\left(\pi^{S}\right)^{\prime} k^{N}\right)-\sum_{S \in \mathcal{B}} r \varepsilon^{S}\left(P^{N}\left(\pi^{S}\right)^{\prime} d^{S}+\delta\left(\pi^{S}\right)^{\prime} k^{N}\right) \geq r c(N) .
$$

Recall the set $\mathcal{B A}$ introduced in the proof of Lemma 1. For each coalition $S \in \mathcal{B}$, consider $l_{S}=r \lambda_{S}^{*}$ replicas of $S$ in the set $\mathcal{B A}$. Therefore, we have in total $m$ coalitions in the new collection (see (1)). We assume an arbitrary ordering of these coalitions so that we can refer, without confusion, to any coalition, say $S_{j}$, by its index in such a sequence. Then, using this set the first term in the left-hand side of (6) can be rewritten as

$$
\begin{gathered}
\sum_{j=1}^{m}\left(P^{N}\left(\pi^{S_{j}}\right)^{\prime} d^{S_{j}}+\delta\left(\pi^{S_{j}}\right)^{\prime} k^{N}\right)=\sum_{j=1}^{m} \sum_{t=1}^{T}\left(P_{t}^{N}\left(\pi^{S_{j}}\right) d_{t}^{S_{j}}+\delta_{t}\left(\pi^{S_{j}}\right) k_{t}^{N}\right) \\
=\sum_{t=1}^{T} \sum_{i=1}^{n} d_{t}^{i} \sum_{\left\{k: S_{j} \ni i\right\}} P_{t}^{N}\left(\pi^{S_{j}}\right)+\sum_{t=1}^{T} \sum_{j=1}^{m} \delta_{t}\left(\pi^{S_{j}}\right) k_{t}^{N} .
\end{gathered}
$$

Now, the expression in (8) is stated as: 


$$
\begin{aligned}
& \sum_{t=1}^{T}\left(\sum_{\substack{i=1 \\
d_{t}^{i}=0}}^{n} d_{t}^{i} \sum_{\left\{k: S_{j} \ni i\right\}} P_{t}^{N}\left(\pi^{S_{j}}\right)+\sum_{\substack{i=1 \\
d_{t}^{i}>0}}^{n} d_{t}^{i} \sum_{\left\{k: S_{j} \ni i\right\}} P_{t}^{N}\left(\pi^{S_{j}}\right)\right) \\
& \quad+\sum_{t=1}^{T} \sum_{j=1}^{m} \delta_{t}\left(\pi^{S_{j}}\right) k_{t}^{N}
\end{aligned}
$$

From the above formula and using the definition in (2) we get:

$$
\begin{aligned}
& \sum_{t=1}^{T} \sum_{i=1}^{n} d_{t}^{i} \sum_{\left\{k: S_{j} \ni i\right\}} P_{t}^{N}\left(\pi^{S_{j}}\right)+\sum_{t=1}^{T} \sum_{j=1}^{m} \delta_{t}\left(\pi^{S_{j}}\right) k_{t}^{N} \geq \sum_{t=1}^{T} \sum_{i=1}^{n} d_{t}^{i} \sum_{j=1}^{r} P_{t}^{N}\left(\psi^{j}\right) \\
& \quad d_{t}^{i}>0 \\
& \quad+\sum_{t=1}^{T} \sum_{j=1}^{r} \delta_{t}\left(\psi^{j}\right) k_{t}^{N}
\end{aligned}
$$

Hence, since $\sum_{i=1}^{n} d_{t}^{i}=d_{t}^{N}$, the right-hand side of (10) equals the following:

$$
\begin{aligned}
& =\sum_{j=1}^{r} \sum_{t=1}^{T}\left(P_{t}^{N}\left(\psi^{j}\right) d_{t}^{N}+\delta_{t}\left(\psi^{j}\right) k_{t}^{N}\right) \\
& \geq \sum_{j=1}^{r} \sum_{t=1}^{T}\left(P_{t}^{N}\left(\sigma^{N}\right) d_{t}^{N}+\delta_{t}\left(\sigma^{N}\right) k_{t}^{N}\right) \\
& =\sum_{j=1}^{r} c(N)=r c(N) .
\end{aligned}
$$

Thus, finally from (7) and (11) we get the following inequality:

$$
\sum_{S \in \mathcal{B}} r \lambda_{S}^{\varepsilon^{S}}\left(P^{N}\left(\pi^{S}\right)^{\prime} d^{S}+\delta\left(\pi^{S}\right)^{\prime} k^{N}\right) \geq r c(N), \forall \lambda_{S}^{\varepsilon^{S}} \text { satisfying }(5) .
$$

Hence, taking limit in (12) when $\lambda_{S}^{\varepsilon_{S}^{S}} \rightarrow \lambda_{S}$ for all $S \in \mathcal{B}$ we obtain:

$$
\sum_{S \in \mathcal{B}} \lambda_{S}\left(P^{N}\left(\pi^{S}\right)^{\prime} d^{S}+\delta\left(\pi^{S}\right)^{\prime} k^{N}\right) \geq c(N)
$$

what concludes the proof.

We note in passing that every subgame of a SI-game is a new SI-game. Thus, Theorem 1 implies that every SI-game is totally balanced and therefore 
subadditive $^{1}$. Moreover, SI-games are neither monotonous (see example 5) nor concave (see Example 4.4 in Guardiola et al. 2009).

Remark 1 From the central part of the proof of Theorem 1, we deduce that the balanced character is ensured for any balanced collection with rational balancing coefficients. Observe that from its definition any set of balancing coefficients of a balanced collection of coalitions has to satisfy the conditions that define the feasible set of the following linear program. Therefore, balancing coefficients are feasible solutions of this problem.

$$
\begin{aligned}
\max & \sum_{S \subset N} \lambda_{S} c(S) \\
\text { s.t. } & \sum_{S: S \ni i} \lambda_{S}=1 \quad i=1, \ldots, n \\
& \lambda_{S} \geq 0 \quad \forall S \subset N .
\end{aligned}
$$

Therefore, since the feasible region of the above problem is rational polytope and thus, it has all its extreme points being rational numbers we deduce that balancedness holds for those choices. In addition, any non rational family of balanced coefficients must be a convex combination of extreme points in this polyhedron. Hence, once balancedness is proved for any rational collection of balancing coefficients, we can apply the following construction to the general case of real coefficients without taking limit of sequences as done in the proof of Theorem 1.

Let $\mathcal{B}$ be a balanced collection with non-rational balancing coefficients $\left\{\lambda_{S}^{B}\right\}_{S \in \mathcal{B}}$. There exist $B^{1}, \ldots, B^{k}$ balanced collections with rational balancing coefficients $\left\{\left\{\lambda_{S}^{B^{1}}\right\}_{S \in B^{1}}, \ldots,\left\{\lambda_{S}^{B^{k}}\right\}_{S \in B^{k}}\right\}$ and $\alpha=\left(\alpha^{1}, \ldots, \alpha^{k}\right) \geq 0, \sum_{i=1}^{k} \alpha^{i}=1$ such that $\mathcal{B}=$ $\bigcup_{i=1}^{k} B^{i}$ and $\lambda_{S}^{B}=\sum_{i=1}^{k} \alpha^{i} \lambda_{S}^{B^{i}}$. (We assume that $\lambda_{S}^{B^{i}}=0$ whenever $S \notin B^{i}$.) Finally,

$$
\sum_{S \in \mathcal{B}} \lambda_{S}^{B} c(S)=\sum_{S \in \mathcal{B}} \sum_{i=1}^{k} \alpha^{i} \lambda_{S}^{B^{i}} c(S)=\sum_{i=1}^{k} \alpha^{i}\left(\sum_{S \in B^{i}} \lambda_{S}^{B^{i}} c(S)\right) \geq c(N) .
$$

In fact, from the above argument we deduce something more general: proving balancedness for collections with rational balancing coefficients suffices.

\section{Extended Owen points}

We have just proven the stability of the grand coalition, in the sense of the core. We know that there always exists a core-allocation for SI-games but we do not know how to construct it. This section is devoted to find sufficient conditions for the extended Owen point to belong to the core.

The Owen point, introduced in Guardiola et al. (2009), is a core-allocation for PIgames which represents the cost that each player has to pay when producing at the minimum operational cost (see also Guardiola et al. (2008)). If we consider a SI-

\footnotetext{
${ }^{1}$ It is well known that every totally balanced cost game is subadditive.
} 
situation $(N, D, Z)$ with $K=0$, that is a PI-situation, the Owen point, $o=\left(o_{i}\right)_{i \in N}$, is given by

$$
o_{i}:=\sum_{t=1}^{T} P_{t}^{N}\left(\sigma^{N}\right) d_{t}^{i}, \quad \text { for all } i \in N
$$

In this section, we introduce a parametric family of cost allocations with the flavor of the Owen point but appropriate to SI-games. We call it the family of extended Owen points. The interested reader is referred to Perea et al. $(2009,2012)$ for alternative extensions of the concept of Owen point. Before defining this new family of cost allocations, we need to introduce some previous concepts.

Let $(N, D, Z)$ be a SI-situation and $(N, c)$ the associated SI-game. We define the reduced SI-situation associated to $(N, D, Z)$ as a SI-situation $(N, D, \widetilde{Z})$ with $\widetilde{Z}:=$ $(\widetilde{K}, \widetilde{H}, \widetilde{B}, \widetilde{P})$ where

$$
\widetilde{K}:=\left[k^{N}, \ldots, k^{N}\right]^{\prime}, \widetilde{H}:=\left[h^{N}, \ldots, h^{N}\right]^{\prime}, \widetilde{B}:=\left[b^{N}, \ldots, b^{N}\right]^{\prime}, \widetilde{P}:=\left[p^{N}, \ldots, p^{N}\right]^{\prime} .
$$

Note that reduced SI-situations are the simplest SI-situations in that all their costs (the lowest possible costs for each period) are the same for all players in all periods.

We denote by $(N, \widetilde{c})$ the cost game associated to the reduced SI-situation $(N, D, \widetilde{Z})$. Notice that $\widetilde{c}(S) \leq c(S)$ for all $S \subset N$ and $\widetilde{c}(N)=c(N)$. Hence $\operatorname{Core}(N, \widetilde{c}) \subseteq \operatorname{Core}(N, c)$. Clearly, each ELS-situation corresponds with a reduced SI-situation for an appropriate choice of parameters since the costs involved in each period are the same for all the players (see Van den Heuvel et al. 2007). Hence, ELS-situations are particular cases of SI-situations.

Next we define the following sets:

- Set of ordering periods: $T^{S}:=\left\{t \in T: \delta_{t}\left(\sigma^{S}\right)=1\right\}$ for all $S \subseteq N$. It is easy to check that,

$$
\sum_{t=1}^{T} \delta_{t}\left(\sigma^{S}\right) k_{t}^{S}=\sum_{t \in T^{S}} k_{t}^{S}
$$

- Set of consolidated periods: $\Upsilon:=\left\{t \in T: \exists i \in N\right.$ such that $\delta_{t}\left(\sigma^{S}\right)=1$ for all $S \subseteq N$ with $i \in S\}$. A period is consolidated if there exists at least one player such that he forces placing an order at this period to any coalition that he belongs to.

We can distinguish two classes of costs for every coalition $S \subseteq N$. Variable costs $P^{S}\left(\sigma^{S}\right)^{\prime} d^{S}$, which depends on demands, and non-consolidated fixed costs $\sum_{t \in T^{S} \backslash \Upsilon} k_{t}^{S}$. Next we define for each $S \subseteq N$

$$
\begin{aligned}
& N(S):=P^{N}\left(\sigma^{N}\right)^{\prime} d^{S}-P^{S}\left(\sigma^{S}\right)^{\prime} d^{S}, \\
& M(S):=\sum_{t \in T^{N} \backslash \Upsilon} k_{t}^{N}-\sum_{t \in T^{S} \backslash \Upsilon} k_{t}^{S} .
\end{aligned}
$$


Notice that $N(S)$ and $M(S)$ represent the difference between the ordering plans $\sigma^{S}$ and $\sigma^{N}$ related to variable and non-consolidated fixed costs.

We are ready now to define the family of extended Owen points.

$$
\left\{\omega(\alpha) \in \mathbb{R}^{N}: \alpha \in \mathbb{R}_{+}^{N} \text { such that } \alpha(N)>0\right\}
$$

where

$$
\omega_{i}(\alpha):=\sum_{t=1}^{T} P_{t}^{N}\left(\sigma^{N}\right) d_{t}^{i}+\sum_{t \in \Upsilon: i \in J_{t}} \frac{k_{t}^{N}}{\left|J_{t}\right|}+\frac{\alpha_{i}}{\alpha(N)} \sum_{t \in T^{N} \backslash \Upsilon} k_{t}^{N}
$$

for all $i \in N$ and $J_{t}:=\left\{i \in N: \delta_{t}\left(\sigma^{i}\right)=1\right\}$.

Notice that the above family of cost allocation is a parametric family depending on $\alpha \in \mathbb{R}_{+}^{N}$ such that $\alpha(N)>0$.

Next proposition shows that, if the optimal ordering plan for the grand coalition reduces variable and non-consolidated fixed costs with respect to any coalition $S \subseteq N$, then the family of extended Owen points is a core-allocation family.

Proposition 1 Let $(N, D, Z)$ a SI-situation and $(N, c)$ the corresponding SI-game. If $N(S), M(S) \leq 0$ for all $S \subseteq N$, then for each $\alpha \in \mathbb{R}_{+}^{N}$ such that $\alpha(N)>0$, the allocation $\omega(\alpha)=\left(\omega_{1}(\alpha), \ldots, \omega_{n}(\alpha)\right)$ defined in (12) is a core-allocation.

Proof Let $(N, D, Z)$ be a SI-situation and $(N, c)$ the corresponding SI-game. Then,

$$
\sum_{i \in S} \omega_{i}(\alpha)=\sum_{t=1}^{T} P_{t}^{N}\left(\sigma^{N}\right) d_{t}^{S}+\sum_{i \in S} \sum_{t \in \Upsilon: i \in J_{t}} \frac{k_{t}^{N}}{\left|J_{t}\right|}+\frac{\alpha(S)}{\alpha(N)} \sum_{t \in T^{N} \backslash \Upsilon} k_{t}^{N}
$$

Note that, $\quad \sum_{i \in S} \sum_{t \in \Upsilon: i \in J_{t}} \frac{k_{t}^{N}}{\left|J_{t}\right|} \leq \sum \quad t \in \Upsilon \quad\left|J_{t}\right| \frac{k_{t}^{S}}{\left|J_{t}\right|} \quad$ since $\quad k_{t}^{N} \leq k_{t}^{S} \quad$ and

$$
\delta_{t}\left(\sigma^{S}\right)=1
$$

$\left|\left\{i \in S: i \in J_{t}\right\}\right| \leq\left|J_{t}\right|$. Therefore (13) is less than or equal to:

$$
\begin{gathered}
\sum_{t=1}^{T} P_{t}^{S}\left(\sigma^{S}\right) d_{t}^{S}+\sum_{\substack{t \in \Upsilon \\
\delta_{t}\left(\sigma^{S}\right)=1}}\left|J_{t}\right| \frac{k_{t}^{S}}{\left|J_{t}\right|}+\frac{\alpha(S)}{\alpha(N)} \sum_{t \in T^{S} \backslash \Upsilon} k_{t}^{S}+\left(N(S)+\frac{\alpha(S)}{\alpha(N)} M(S)\right) \\
\leq \sum_{t=1}^{T} P_{t}^{S}\left(\sigma^{S}\right) d_{t}^{S}+\sum_{t=1}^{T} \delta_{t}\left(\sigma^{S}\right) k_{t}^{S}=c(S)
\end{gathered}
$$

It is easy to check that $\omega$ is efficient. Hence, $\omega \in \operatorname{Core}(N, c)$.

The above result is illustrated in the next two examples. 
Example 2 Consider the following SI-situation with three periods and two players:

\begin{tabular}{llllllllllllllll}
\hline & \multicolumn{1}{c}{ Demand } & & \multicolumn{1}{c}{ Production } & \multicolumn{3}{c}{ Inventory } & \multicolumn{3}{c}{ Backlogging } & \multicolumn{3}{c}{ Setup } \\
\hline$P 1$ & 2 & 0 & 0 & 1 & 1 & 1 & 1 & 1 & 1 & 6 & 6 & 1 & 5 & 5 & 1 \\
$P 2$ & 0 & 2 & 2 & 1 & 1 & 1 & 0 & 0 & 1 & 0 & 0 & 1 & 5 & 5 & 5 \\
\hline
\end{tabular}

The corresponding SI-game is given by:

\begin{tabular}{lcccccccccccccccc}
\hline & $d_{1}^{s}$ & $d_{2}^{s}$ & $d_{3}^{s}$ & $p_{1}^{s}$ & $p_{2}^{s}$ & $p_{3}^{s}$ & $h_{1}^{s}$ & $h_{2}^{s}$ & $h_{3}^{s}$ & $b_{1}^{s}$ & $b_{2}^{s}$ & $b_{3}^{s}$ & $k_{1}^{s}$ & $k_{2}^{s}$ & $k_{3}^{s}$ & $c(S)$ \\
\hline$\{1\}$ & 2 & 0 & 0 & 1 & 1 & 1 & 1 & 1 & 1 & 6 & 6 & 1 & 5 & 5 & 1 & 7 \\
$\{2\}$ & 0 & 2 & 2 & 1 & 1 & 1 & 0 & 0 & 1 & 0 & 0 & 1 & 5 & 5 & 5 & 9 \\
$\{1,2\}$ & 2 & 2 & 2 & 1 & 1 & 1 & 0 & 0 & 1 & 0 & 0 & 1 & 5 & 5 & 1 & 7 \\
\hline
\end{tabular}

Next table shows the optimal ordering plans, the corresponding cost-plan vectors, and the differences between the ordering plans:

\begin{tabular}{lllllllll}
\hline & $\sigma_{1}^{S}$ & $\sigma_{2}^{S}$ & $\sigma_{3}^{S}$ & $P_{1}^{S}\left(\sigma^{S}\right)$ & $P_{2}^{S}\left(\sigma^{S}\right)$ & $P_{3}^{S}\left(\sigma^{S}\right)$ & $N(S)$ & $M(S)$ \\
\hline$\{1\}$ & 1 & 0 & 0 & 1 & 0 & 0 & 0 & -4 \\
$\{2\}$ & 0 & 2 & 2 & 0 & 1 & 1 & 0 & -4 \\
$\{1,2\}$ & 3 & 3 & 3 & 1 & 1 & 1 & 0 & 0 \\
\hline
\end{tabular}

Since $N(S), M(S) \leq 0$ for all $S \subseteq N$, we can conclude that

$$
\left\{(2,4)+\frac{1}{\alpha(N)}\left(\alpha_{1}, \alpha_{2}\right): \begin{array}{l}
\alpha_{i} \in \mathbb{R}_{+} \forall i \in N \\
\text { with } \alpha(N)>0
\end{array}\right\} \subseteq \operatorname{Core}(N, c) .
$$

The following example shows that the family of extended Owen points, at times, can be a singleton.

Example 3 Consider the following SI-situation with three periods and three players:

\begin{tabular}{|c|c|c|c|c|c|c|c|c|c|c|c|c|c|c|c|}
\hline & \multicolumn{3}{|c|}{ Demand } & \multicolumn{3}{|c|}{ Production } & \multicolumn{3}{|c|}{ Inventory } & \multicolumn{3}{|c|}{ Backlogging } & \multicolumn{3}{|c|}{ Setup } \\
\hline$P 1$ & 6 & 5 & 2 & 3 & 1 & 1 & 2 & 3 & 3 & 1 & 3 & 1 & 4 & 3 & 4 \\
\hline$P 2$ & 4 & 1 & 1 & 5 & 1 & 4 & 2 & 1 & 3 & 1 & 3 & 1 & 0 & 2 & 5 \\
\hline$P 3$ & 1 & 4 & 1 & 2 & 1 & 3 & 3 & 1 & 1 & 1 & 3 & 1 & 0 & 0 & 5 \\
\hline
\end{tabular}


The corresponding SI-game is given by:

\begin{tabular}{lllllllllllllllll}
\hline & $d_{1}^{s}$ & $d_{2}^{s}$ & $d_{3}^{s}$ & $p_{1}^{s}$ & $p_{2}^{s}$ & $p_{3}^{s}$ & $h_{1}^{s}$ & $h_{2}^{s}$ & $h_{3}^{s}$ & $b_{1}^{s}$ & $b_{2}^{s}$ & $b_{3}^{s}$ & $k_{1}^{s}$ & $k_{2}^{s}$ & $k_{3}^{s}$ & $c(S)$ \\
\hline$\{1\}$ & 6 & 5 & 2 & 3 & 1 & 1 & 2 & 3 & 3 & 1 & 3 & 1 & 4 & 3 & 4 & 36 \\
$\{2\}$ & 4 & 1 & 1 & 5 & 1 & 4 & 2 & 1 & 3 & 1 & 3 & 1 & 0 & 2 & 5 & 21 \\
$\{3\}$ & 1 & 4 & 1 & 2 & 1 & 3 & 3 & 1 & 1 & 1 & 3 & 1 & 0 & 0 & 5 & 8 \\
$\{1,2\}$ & 10 & 6 & 3 & 3 & 1 & 1 & 2 & 1 & 3 & 1 & 3 & 1 & 0 & 2 & 4 & 44 \\
$\{1,3\}$ & 7 & 9 & 3 & 2 & 1 & 1 & 2 & 1 & 1 & 1 & 3 & 1 & 0 & 0 & 4 & 29 \\
$\{2,3\}$ & 5 & 5 & 2 & 2 & 1 & 3 & 2 & 1 & 1 & 1 & 3 & 1 & 0 & 0 & 5 & 19 \\
$\{1,2,3\}$ & 11 & 10 & 4 & 2 & 1 & 1 & 2 & 1 & 1 & 1 & 3 & 1 & 0 & 0 & 4 & 40 \\
\hline
\end{tabular}

Next table shows the optimal ordering plans, the corresponding cost-plan vectors, and the differences between the ordering plans:

\begin{tabular}{lllllllll}
\hline & $\sigma_{1}^{S}$ & $\sigma_{2}^{S}$ & $\sigma_{3}^{S}$ & $P_{1}^{S}\left(\sigma^{S}\right)$ & $P_{2}^{S}\left(\sigma^{S}\right)$ & $P_{3}^{S}\left(\sigma^{S}\right)$ & $N(S)$ & $M(S)$ \\
\hline$\{1\}$ & 1 & 2 & 3 & 3 & 1 & 1 & -4 & -4 \\
$\{2\}$ & 2 & 2 & 2 & 4 & 1 & 2 & -8 & 0 \\
$\{3\}$ & 1 & 2 & 2 & 2 & 1 & 2 & 0 & 0 \\
$\{1,2\}$ & 1 & 2 & 2 & 3 & 1 & 2 & -10 & 0 \\
$\{1,3\}$ & 1 & 2 & 2 & 2 & 1 & 2 & 0 & 0 \\
$\{2,3\}$ & 1 & 2 & 2 & 2 & 1 & 2 & 0 & 0 \\
$\{1,2,3\}$ & 1 & 2 & 2 & 2 & 1 & 2 & 0 & 0 \\
\hline
\end{tabular}

Since $N(S), M(S) \leq 0$ for all $S \subseteq N$, we can conclude that

$$
\left\{(21,11,8)+\frac{0}{\alpha(N)}\left(\alpha_{1}, \alpha_{2}, \alpha_{3}\right): \begin{array}{l}
\alpha_{i} \in \mathbb{R}_{+} \forall i \in N \\
\text { with } \alpha(N)>0
\end{array}\right\} \subseteq \operatorname{Core}(N, c) .
$$

If we compare the Extended Owen point with other well-known solutions for TU games as Shapley value (Shapley 1953) and nucleolus (Schmeidler 1969) some differences arise. First of all, $\omega(\alpha)$ is not a game-theoretical solution, that is, if we consider two different SI-situations that produce the same game, both the Shapley value and the nucleolus will give the same solution. However, $\omega(\alpha)$ is not a gametheoretical solution since its definition only applies on SI-situations. On the other hand, the Extended Owen point, although it is not taking into account all the contributions of the players, intend to allocate the costs in an equitable way: getting the players to produce their demand at the lowest cost, distributing the setup costs of 
the consolidated periods equitably between the players who use them and considering all possible ways to distribute the remaining setup cost. In addition, the Extended Owen point, like the Shapley value, belongs to core under certain conditions, and it is much easier to calculate than the nucleolus.

A weaker sufficient condition to ensure the cost allocation $\omega(\alpha)$ to be in the core is given by the following corollary.

Corollary 1 Let $(N, D, Z)$ a SI-situation and $(N, c)$ the corresponding SI-game. If there exists $\alpha \in \mathbb{R}_{+}^{N}$ such that $\alpha(N)>0$ and $N(S)+\frac{\alpha(S)}{\alpha(N)} M(S) \leq 0$ for all $S \subseteq N$, then $\omega(\alpha)$ is a core-allocation.

The example below illustrates the above condition.

Example 4 Consider the SI-situation described by the following table.

\begin{tabular}{llllllllllllllll}
\hline & \multicolumn{1}{c}{ Demand } & \multicolumn{3}{c}{ Production } & \multicolumn{3}{c}{ Inventory } & \multicolumn{3}{c}{ Backlogging } & \multicolumn{3}{c}{ Setup } \\
\hline$P 1$ & 5 & 5 & 2 & 3 & 1 & 1 & 2 & 3 & 3 & 1 & 2 & 1 & 3 & 1 & 2 \\
$P 2$ & 4 & 1 & 1 & 5 & 1 & 4 & 2 & 1 & 3 & 1 & 2 & 1 & 3 & 1 & 2 \\
$P 3$ & 1 & 4 & 5 & 2 & 1 & 3 & 3 & 2 & 1 & 1 & 2 & 1 & 2 & 1 & 2 \\
\hline
\end{tabular}

The corresponding SI-game is shown in the next table:

\begin{tabular}{lcccccccccccccccc}
\hline & $d_{1}^{s}$ & $d_{2}^{s}$ & $d_{3}^{s}$ & $p_{1}^{s}$ & $p_{2}^{s}$ & $p_{3}^{s}$ & $h_{1}^{s}$ & $h_{2}^{s}$ & $h_{3}^{s}$ & $b_{1}^{s}$ & $b_{2}^{s}$ & $b_{3}^{s}$ & $k_{1}^{s}$ & $k_{2}^{s}$ & $k_{3}^{s}$ & $c$ \\
\hline$\{1\}$ & 5 & 5 & 2 & 3 & 1 & 1 & 2 & 3 & 3 & 1 & 2 & 1 & 3 & 1 & 2 & 25 \\
$\{2\}$ & 4 & 1 & 1 & 5 & 1 & 4 & 2 & 1 & 3 & 1 & 2 & 1 & 3 & 1 & 2 & 16 \\
$\{3\}$ & 1 & 4 & 5 & 2 & 1 & 3 & 3 & 2 & 1 & 1 & 2 & 1 & 2 & 1 & 2 & 23 \\
$\{1,2\}$ & 9 & 6 & 3 & 3 & 1 & 1 & 2 & 1 & 3 & 1 & 2 & 1 & 3 & 1 & 2 & 39 \\
$\{1,3\}$ & 6 & 9 & 7 & 2 & 1 & 1 & 2 & 2 & 1 & 1 & 2 & 1 & 2 & 1 & 2 & 33 \\
$\{2,3\}$ & 5 & 5 & 6 & 2 & 1 & 3 & 2 & 1 & 1 & 1 & 2 & 1 & 2 & 1 & 2 & 30 \\
$\{1,2,3\}$ & 10 & 10 & 8 & 2 & 1 & 1 & 2 & 1 & 1 & 1 & 2 & 1 & 2 & 1 & 2 & 43 \\
\hline
\end{tabular}

The optimal ordering plans, the corresponding cost-plan vectors, and the differences between the ordering plans can be found in the last table:

\begin{tabular}{lllllllll}
\hline & $\sigma_{1}^{S}$ & $\sigma_{2}^{S}$ & $\sigma_{3}^{S}$ & $P_{1}^{S}\left(\sigma^{S}\right)$ & $P_{2}^{S}\left(\sigma^{S}\right)$ & $P_{3}^{S}\left(\sigma^{S}\right)$ & $N(S)$ & $M(S)$ \\
\hline$\{1\}$ & 2 & 2 & 3 & 3 & 1 & 1 & -5 & 2 \\
$\{2\}$ & 2 & 2 & 2 & 3 & 1 & 2 & -5 & 2 \\
$\{3\}$ & 2 & 2 & 2 & 3 & 1 & 3 & -11 & 2 \\
$\{1,2\}$ & 2 & 2 & 3 & 3 & 1 & 1 & -9 & 2 \\
$\{1,3\}$ & 1 & 2 & 3 & 2 & 1 & 1 & 0 & 0 \\
$\{2,3\}$ & 1 & 2 & 2 & 2 & 1 & 2 & -6 & 0 \\
$\{1,2,3\}$ & 1 & 2 & 3 & 2 & 1 & 1 & 0 & 0 \\
\hline
\end{tabular}


It can be easily checked that $N(S)+\frac{\alpha(S)}{\alpha(N)} M(S) \leq 0$ for all $\alpha \in \mathbb{R}_{+}^{N}$ such that $\alpha(N)>0$ and all $S \subseteq N$. Hence

$$
\left\{\left(\frac{58}{3}, \frac{31}{3}, \frac{34}{3}\right)+\frac{2}{\alpha(N)}\left(\alpha_{1}, \alpha_{2}, \alpha_{3}\right): \begin{array}{c}
\alpha_{i} \in \mathbb{R}_{+} \forall i \in N \\
\text { with } \alpha(N)>0
\end{array}\right\} \subseteq \operatorname{Core}(N, c) .
$$

We finish this section with a simpler sufficient condition to check core membership.

Corollary 2 Let $(N, D, Z)$ be a SI-situation and $(N, c)$ the corresponding SI-game. If the reduced SI-situation $(N, D, \widetilde{Z})$ satisfies one of the following conditions:

(i) $\tilde{N}(S), \widetilde{M}(S) \leq 0$,

(ii) there exists $\alpha \in \mathbb{R}_{+}^{N}$ such that $\alpha(N)>0$ and $\widetilde{N}(S)+\frac{\alpha(S)}{\alpha(N)} \widetilde{M}(S) \leq 0$ for all $S \subseteq N$

where $\widetilde{N}(S), \widetilde{M}(S)$ are the corresponding values for the reduced SI-situation for all $S \subseteq N$, then $\omega(\alpha)$ is a core-allocation.

\section{Consolidated situations and stability}

We focus now on those SI-situations that are consolidated. Then we analyze their effect on the stability of the extended Owen points.

To do that, we recall the concept of consolidated period: a period $t$ is consolidated if for some player that would order in $t$ any coalition where he is included would also place an order in that period. This leads to the definition of consolidated situation. A SI-situation is consolidated if all the periods where any coalition places an order are consolidated. This idea of consolidation is a refinement of the original scheme that makes coalitions perform like some of their players. The concept is formalized in the following definition.

Definition 1 A SI-situation $(N, D, Z)$ is consolidated if $T^{S} \subseteq \Upsilon$ for all $S \subseteq N$.

From the above definition, it is clear that in any consolidated SI-situation, $M(S)=0$ for any coalition $S \subseteq N$, since $\sum_{t \in T^{S} \backslash \curlyvee} k_{t}^{S}=0$. Based in this fact, we can provide an extended Owen point in the core for consolidated SI-games. The following technical lemma is needed to prove this result.

Lemma 2 Let $(N, D, Z)$ be a consolidated SI-situation and $(N, c)$ the corresponding SI-game. Then $P_{t}^{S}\left(\sigma^{S}\right) \geq P_{t}^{R}\left(\sigma^{R}\right)$ for all $t \in T$ with $d_{t}^{S} \neq 0$ and for all $S \subseteq R \subseteq N$.

Proof Suppose that $\exists t^{\prime} \in T$ with $d_{t^{\prime}}^{S} \neq 0$ such that $P_{t^{\prime}}^{S}\left(\sigma^{S}\right)<P_{t^{\prime}}^{R}\left(\sigma^{R}\right)$ then $\sigma_{t^{\prime}}^{S}=r$ and $\sigma_{t^{\prime}}^{R}=r^{\prime}$ with $r \neq r^{\prime} . \quad \delta_{r}\left(\sigma^{S}\right)=1$ therefore $\delta_{r}\left(\sigma^{R}\right)=1$ since $(N, D, Z)$ is a consolidated SI-situation. If we take the next feasible plan, 


$$
\sigma^{*}:=\left\{\begin{array}{cc}
\sigma_{t}^{R} & \text { if } t \neq t^{\prime}, \\
r & \text { if } t=t^{\prime},
\end{array}\right.
$$

then $P_{t^{\prime}}^{R}\left(\sigma^{*}\right) \leq P_{t^{\prime}}^{S}\left(\sigma^{S}\right)<P_{t^{\prime}}^{R}\left(\sigma^{R}\right)$ and $\delta\left(\sigma^{*}\right)^{\prime} k^{R} \leq \delta\left(\sigma^{R}\right)^{\prime} k^{R}$. Hence,

$$
c(R)=P^{R}\left(\sigma^{R}\right)^{\prime} d^{R}+\delta\left(\sigma^{R}\right)^{\prime} k^{R}>P^{R}\left(\sigma^{*}\right)^{\prime} d^{R}+\delta\left(\sigma^{*}\right)^{\prime} k^{R},
$$

and this is a contradiction because $\sigma^{R}$ is an optimal ordering plan of coalition $R \subseteq N$.

Note that the above lemma exhibits a monotonicity property with respect to the ordering policies. We mean that, the smaller the coalition, the greater the cost of satisfying demand in each single period.

Proposition 2 Let $(N, D, Z)$ be a consolidated SI-situation and $(N, c)$ the asociated SI-game. Then, the allocation $\psi \in \mathbb{R}^{N}$ given by

$$
\psi_{i}:=\sum_{t=1}^{T} P_{t}^{N}\left(\sigma^{N}\right) d_{t}^{i}+\sum_{t \in \Upsilon: i \in J_{t}} \frac{k_{t}^{N}}{\left|J_{t}\right|},
$$

for all $i \in N$ where $J_{t}:=\left\{i \in N: \delta_{t}\left(\sigma^{i}\right)=1\right\}$, is a core-allocation.

Proof We suppose that $(N, D, Z)$ is consolidated. Note that if $T^{S} \subseteq \Upsilon$ for $S \subseteq N$ then $M(S)=0$ and $\sum_{t \in T^{N} \backslash \Upsilon} k_{t}^{N}=0$. We know $\sigma_{t}^{S} \in \Upsilon$ for all $t \in T$ and for all $S \subseteq N$ with $d_{t}^{S} \neq 0$, then by Lemma , $P^{S}\left(\sigma^{S}\right) \geq P^{N}\left(\sigma^{N}\right)$. Hence $N(S) \leq 0$ for all $S \subseteq N$, By Proposition $1 \psi$ is a core-allocation.

Corollary 3 Let $(N, D, Z)$ be a SI-situation and $(N, c)$ the corresponding SI-game. If the reduced SI-situation $(N, D, \widetilde{Z})$ is consolidated, then $\psi \in \operatorname{Core}(N, c)$.

From now on, the allocation $\psi$ will be called the extended Owen point for consolidated SI-games (those which come from consolidated SI-situations). The reader may notice that $\psi=\omega(\alpha)$ for all $\alpha \in \mathbb{R}_{+}^{N}$.

Recall that according to Sprumont (1990), a population monotonic allocation scheme (pmas), for the game $(N, c)$ is a collection of vectors $y^{S} \in \mathbb{R}^{s}$ for all $S \subseteq$ $N, S \neq \varnothing$ such that $y^{S}(S)=c(S)$ for all $S \subseteq N, S \neq \varnothing$, and $y_{i}^{S} \geq y_{i}^{T}$ for all $S \subseteq$ $T \subseteq N$ and $i \in S$. The reader may note that whenever $\left(y^{S}\right)_{\varnothing \neq S \subseteq N}$ is a pmas for $(N, c)$, then $y^{S}$ is a core allocation for the game $\left(S, c_{s}\right)$ for all $S \subseteq N, S \neq \varnothing$. Thus, cost allocations attainded through a pmas are a refinement of the core. This implies that every cost TU game with a pmas is totally balanced but the reciprocal is not true and there are many totally balanced cost TU games without pmas. A core-allocation for $(N, c)$, i.e. $x \in \operatorname{Core}(N, c)$, is reached through a pmas if there exists $\left(y^{S}\right) \varnothing \neq S \subseteq N$ for the game $(N, c)$ such that $y_{i}^{N}=x_{i}$ for all $i \in N$.

The final result of the section explicitly constructs a pmas that realizes the extended Owen point for consolidated SI-games. 
Theorem 2 Let $(N, D, Z)$ be a consolidated SI-situation and $(N, c)$ the corresponding SI-game. Then, $\psi$ can be realized through a pmas.

Proof Define for all $i \in S, S \subseteq N$ and $S \neq \varnothing$,

$$
y_{i}^{S}:=\sum_{t=1}^{T} P_{t}^{S}\left(\sigma^{S}\right) d_{t}^{i}+\sum_{t \in \Upsilon^{S}: i \in J_{t}^{S}} \frac{k_{t}^{S}}{\mid J_{t}^{S \mid}} .
$$

where $\Upsilon^{S}:=\left\{t \in T: \exists i \in S\right.$ such that $\delta_{t}\left(\sigma^{S}\right)=1 \quad$ for all $S \subseteq N$ with $\left.i \in S\right\}$ and $J_{t}^{S}:=\left\{i \in S: \delta_{t}\left(\sigma^{i}\right)=1\right\}$. Then for all $S \subseteq N, S \neq \varnothing$

$$
\sum_{i \in S} y_{i}^{S}=\sum_{t=1}^{T} P_{t}^{S}\left(\sigma^{S}\right) d_{t}^{S}+\sum_{t \in T^{S}} k_{t}^{S}=c(S),
$$

and for all $S \subseteq R \subseteq N, S, R \neq \varnothing$ and for all $i \in S$,

$$
\begin{aligned}
y_{i}^{S} & =\sum_{t=1}^{T} P_{t}^{S}\left(\sigma^{S}\right) d_{t}^{i}+\sum_{t \in \Upsilon^{S}: i \in J_{t}} \frac{k_{t}^{S}}{\left|J_{t}^{S}\right|} \geq \sum_{t=1}^{T} P_{t}^{R}\left(\sigma^{R}\right) d_{t}^{i}+\sum_{t \in \Upsilon^{S}: i \in J_{t}^{S}} \frac{k_{t}^{R}}{\left|J_{t}^{S}\right|} \\
& \geq \sum_{t=1}^{T} P_{t}^{R}\left(\sigma^{R}\right) d_{t}^{i}+\sum_{t \in \Upsilon^{R}: i \in J_{t}^{R}} \frac{k_{t}^{R}}{\left|J_{t}^{R}\right|}=y_{i}^{R},
\end{aligned}
$$

since $\Upsilon^{R} \subseteq \Upsilon^{S}$ and $\left|J_{t}^{S}\right| \leq\left|J_{t}^{R}\right|$ for all $t \in T$.

Finally, we see that $y_{i}^{N}=\psi_{i}$ for all $i \in N$. So, the extended Owen point for consolidated SI-situations $\psi$ can be reached through the pmas $\left(y^{S}\right)_{\varnothing \neq S \subseteq N}$.

From the proof of the above theorem we deduce that for every consolidated SIgame, a pmas can be built just taking the extended Owen point for each subgame and gathering them all as a collection of vectors. Notice that this construction shows a strong consistency, in terms of stability, of this point solution.

\begin{tabular}{|c|c|c|c|c|c|c|c|c|c|c|c|c|c|c|c|}
\hline \multirow[b]{2}{*}{$P 1$} & \multicolumn{3}{|c|}{ Demand } & \multicolumn{3}{|c|}{ Production } & \multicolumn{3}{|c|}{ Inventory } & \multicolumn{3}{|c|}{ Backlogging } & \multicolumn{3}{|c|}{ Setup } \\
\hline & 1 & 3 & 1 & 1 & 1 & 1 & 1 & 1 & 1 & 1 & 1 & 1 & 1 & 1 & 5 \\
\hline$P 2$ & 2 & 1 & 1 & 2 & 3 & 4 & 1 & 1 & 1 & 1 & 1 & 1 & 1 & 1 & 5 \\
\hline$P 3$ & 2 & 1 & 3 & 2 & 3 & 5 & 1 & 1 & 1 & 1 & 1 & 1 & 1 & 1 & 5 \\
\hline
\end{tabular}

The final example illustrates all the above mentioned results. In addition, it shows that the core of consolidated SI-games is not necessarily a singleton.

Example 5 Consider the following SI-situation with three periods and three players: 
The corresponding SI-game is given in the next table:

\begin{tabular}{lcccccccccccccccc}
\hline & $d_{1}^{s}$ & $d_{2}^{s}$ & $d_{3}^{s}$ & $p_{1}^{s}$ & $p_{2}^{s}$ & $p_{3}^{s}$ & $h_{1}^{s}$ & $h_{2}^{s}$ & $h_{3}^{s}$ & $b_{1}^{s}$ & $b_{2}^{s}$ & $b_{3}^{s}$ & $k_{1}^{s}$ & $k_{2}^{s}$ & $k_{3}^{s}$ & $c$ \\
\hline$\{1\}$ & 1 & 3 & 1 & 1 & 1 & 1 & 1 & 1 & 1 & 1 & 1 & 1 & 1 & 1 & 5 & 8 \\
$\{2\}$ & 2 & 1 & 1 & 2 & 3 & 4 & 1 & 1 & 1 & 1 & 1 & 1 & 1 & 1 & 5 & 12 \\
$\{3\}$ & 2 & 1 & 3 & 2 & 3 & 5 & 1 & 1 & 1 & 1 & 1 & 1 & 1 & 1 & 5 & 20 \\
$\{1,2\}$ & 3 & 4 & 2 & 1 & 1 & 1 & 1 & 1 & 1 & 1 & 1 & 1 & 1 & 1 & 5 & 13 \\
$\{1,3\}$ & 3 & 4 & 4 & 1 & 1 & 1 & 1 & 1 & 1 & 1 & 1 & 1 & 1 & 1 & 5 & 17 \\
$\{2,3\}$ & 4 & 2 & 4 & 2 & 3 & 4 & 1 & 1 & 1 & 1 & 1 & 1 & 1 & 1 & 5 & 31 \\
$\{1,2,3\}$ & 5 & 4 & 5 & 1 & 1 & 1 & 1 & 1 & 1 & 1 & 1 & 1 & 1 & 1 & 5 & 22 \\
\hline
\end{tabular}

The reader may notice that it comes from a consolidate SI-situation since

\begin{tabular}{llllllll}
\hline & $\sigma_{1}^{S}$ & $\sigma_{2}^{S}$ & $\sigma_{3}^{S}$ & $P_{1}^{S}\left(\sigma^{S}\right)$ & $P_{2}^{S}\left(\sigma^{S}\right)$ & $P_{3}^{S}\left(\sigma^{S}\right)$ & $\delta\left(\sigma^{S}\right)^{\prime} k^{S}$ \\
\hline$\{1\}$ & 2 & 2 & 2 & 2 & 1 & 2 & 1 \\
$\{2\}$ & 1 & 1 & 1 & 2 & 3 & 4 & 1 \\
$\{3\}$ & 1 & 1 & 1 & 2 & 3 & 4 & 1 \\
$\{1,2\}$ & 1 & 2 & 2 & 1 & 1 & 2 & 2 \\
$\{1,3\}$ & 1 & 2 & 2 & 1 & 1 & 2 & 2 \\
$\{2,3\}$ & 1 & 1 & 1 & 2 & 3 & 4 & 1 \\
$\{1,2,3\}$ & 1 & 2 & 2 & 1 & 1 & 2 & 2 \\
\hline
\end{tabular}

The extended Owen point for the above consolidated SI-game is $\psi=\left(7, \frac{11}{2}, \frac{19}{2}\right)$. However, the core of this game does not reduce to it since also $x=(7,5,10) \in$ Core $(N, c)$. In addition, the extended Owen point can be reached through the pmas

$$
\left((8)^{\{1\}},(12)^{\{2\}},(20)^{\{3\}},(7,6)^{\{1,2\}},(7,10)^{\{1,3\}},\left(\frac{23}{2}, \frac{39}{2}\right)^{\{2,3\}},\left(7, \frac{11}{2}, \frac{19}{2}\right)^{\{1,2,3\}}\right) \text {. }
$$

\section{Concluding remarks}

Cooperation in periodic review finite horizon inventory models has been already analyzed in Guardiola et al. (2008, 2009) and Van den Heuvel et al. (2007). This paper extends previous approaches in the literature considering a more general model that includes non-homogeneous set up and backlogging costs. We prove that this model of cooperation, by sharing technologies for the production, carrying of goods and distribution channels, induces savings because the resulting game is totally balanced. Moreover, we have introduced a parametric family of allocations based on the Owen point (see Guardiola et al. 2008, 2009) and a subclass of games that enjoys a population monotonic allocation scheme. 
The stability property of the above mentioned mode of coordination leads us to mention two related future research lines: (1) analyzing the cooperation aspects of broader subclasses of inventory situations for which it is possible to provide explicit solutions; and (2) studying the relationships between the cores that arise from situations with and without set up costs.

Acknowledgements The research of the second author is partially supported Financial support of the Ministerio de Ciencia, Innovación y Universidades (MCIU), the Agencia Estatal de Investigación (AEI) and the Fondo Europeo de Desarrollo Regional (FEDER) under the project PGC2018-097965-B-I00. The research of the third author has been partially supported by Spanish Ministry of Education and Science/ FEDER grant number MTM2016-74983-C02-01, and projects FEDER-US-1256951, P18-FR-1422 Junta de Andalucia, CEI-3-FQM331 and NetmeetData: Ayudas Fundación BBVA a equipos de investigación científica 2019.

Funding Open Access funding provided thanks to the CRUE-CSIC agreement with Springer Nature.

Open Access This article is licensed under a Creative Commons Attribution 4.0 International License, which permits use, sharing, adaptation, distribution and reproduction in any medium or format, as long as you give appropriate credit to the original author(s) and the source, provide a link to the Creative Commons licence, and indicate if changes were made. The images or other third party material in this article are included in the article's Creative Commons licence, unless indicated otherwise in a credit line to the material. If material is not included in the article's Creative Commons licence and your intended use is not permitted by statutory regulation or exceeds the permitted use, you will need to obtain permission directly from the copyright holder. To view a copy of this licence, visit http:// creativecommons.org/licenses/by/4.0/.

\section{References}

Bondareva ON (1963) Some applications of linear programming methods to the theory of cooperative games. Probl Kibern 10:119-139 (in Russian)

Borm PEM, Hamers H, Hendrickx R (2001) Operations research games: a survey. TOP 9:139-216

Chen X, Zhang J (2016) Duality approaches to economic lot sizing games. Prod oper Manag 25(7):1203-1215

Dreschel J (2010) Cooperative lot sizing games in supply chains. Springer, Berlin

Feng H, Zeng Y, Cai X, Qian Q, Zhou Y (2021) Altruistic profit allocation rules for joint replenishment with carbon cap-and-trade policy. Eur J Oper Res 290:956-967

Gellekom JRG, Potters JAM, Reijnierse JH, Engel MC, Tijs SH (2000) Characterization of the Owen set of linear production processes. Games Econ Behav 32:139-156

Gopaladesikan M, Uhan NA (2011) Cost sharing for the economic lot-sizing problem with remanufacturing options. Purdue University, optimization-on-line.org/DB\_FILE/2010/09/2733

Guardiola LA, Meca A, Puerto J (2008) PI-games and pmas games: characterizations of Owen point. Math Soc Sci 56:96-108

Guardiola LA, Meca A, Puerto J (2009) Production-Inventory games: a new class of totally balanced combinatorial optimization games. Games Econ Behav 65:205-219

Guardiola LA, Meca A, Puerto J (2021a) Enforcing fair cooperation in production-inventory settings with heterogeneous agents. Ann Oper Res. https://doi.org/10.1007/s10479-021-04083-3

Guardiola LA, Meca A, Puerto J (2021b) Unitary owen points in cooperative lot-sizing models with backlogging. Mathematics 9:869. https://doi.org/10.3390/math9080869

Johnson LA, Montgomery DC (1974) Operations research in production planning, scheduling, and inventory control. John Wiley \& Sons, New York

Karimi B, Ghomi SMTF, Wilson JM (2003) The capacitated lot sizing problem: a review of models and algorithms. Omega 31:365-378 
Li GD, Du DL, Xu DC, Zhang RY (2014) A cost-sharing method for the multi-level economic lot-sizing game. Sci China Inform Sci 57:1-9

Margaretha Gansterer M, Hartl RF (2020) The collaborative multi-level lot-sizing problem with cost synergies. Int J Prod Res 58(2):332-349

Masheli A, Mohammaditabar D (2017) A game theoretic model for capacity-constrained supplier selection by considering joint shipment. J Ind Eng Res Prod Syst 5(10):83-97

Owen G (1975) On the core of linear production games. Math Program 9:358-370

Perea F, Puerto J, Fernández FR (2009) Modeling cooperation on a class of distribution problems. Eur J Oper Res 198(3):726-733

Perea F, Puerto J, Fernández FR (2012) Avoiding unfairness of Owen allocations in linear production processes. Eur J Oper Res 220:125-131

Schmeidler D (1969) The nucleolus of a characteristic function game. SIAM J Appl Math 17:1163-1170

Shapley LS (1953) A value for n-person games. In: Kuhn H, Tucker AW (eds) Contributions to the theory of games II. Princeton University Press, Princeton, pp 307-317

Shapley LS (1967) On balanced sets and cores. Naval Res Logist 14:453-460

Shapley LS, Shubik M (1969) On market games. J Econ Theory 1:9-25

Sprumont Y (1990) Population monotonic allocation schemes for cooperative games with transferable utility. Games Econ Behav 2:378-394

Tamir A (1992) On the complexity of some classes of location problems. Transp Sci 26:352-354

Toriello A, Uhan NA (2014) Dynamic cost allocation for economic lot sizing games. Oper Res Lett 42(1):82-84

Tsao YC, Chen TH, Wu PY (2013) Effects of lot-sizing integration and learning effect on managing imperfect items in a manufacturer-retailer chain. J Appl Math 9:1-11

Van den Heuvel W, Borm P, Hamers H (2007) Economic lot-sizing games. Eur J Oper Res 176:1117-1130

Xu D, Yang R (2009) A cost-sharing method for an economic lot-sizing game. Oper Res Lett 37:107-110

Zangwill WI (1969) A backlogging model and multi-echelon model of a dynamic economic lot size production system- a network approach. Manag Sci 15(9):506-527

Zeng Y, Li J, Cai X (2011) Economic lot-sizing games with perishable inventory. ICSSSM11, Tianjin, pp 1-5. https://doi.org/10.1109/ICSSSM.2011.5959533.

Publisher's Note Springer Nature remains neutral with regard to jurisdictional claims in published maps and institutional affiliations. 